Céline Mavrot

\title{
Compte-rendu de la conférence « The COVID-19 crisis - Lessons for the Courts »
}

\section{European Group for Public Administration}

Catégories d'articles : Comptes rendus de colloque 
[1] La conférence «The COVID-19 crisis - Lessons for the Courts», tenue en ligne le 3 septembre 2020, a proposé un bilan intermédiaire de la réaction des systèmes judiciaires à la crise sanitaire suite à la première vague de l'épidémie de Covid-19 qui a touché la planète avec une ampleur sans précédent à partir de février 2020. Elle a offert un aperçu de l'état de la situation dans plus d'une dizaine de pays sous des perspectives variées, en abordant les répercussions, procédurales, technologiques, politiques et constitutionnelles de la crise. La conférence était organisée par le Groupe d'étude permanent XVIII «Administration de la justice et des tribunaux » du Groupe Européen pour l'Administration Publique (European Group for Public Administration - EGPA). Elle était abritée par le KPM Center for Public Management de l'Université de Berne, présidée par Andreas Lienhard (Université de Berne, Suisse), Daniel Kettiger (Université de Berne), Anne Sanders (Université de Bielefeld, Allemagne) et Marco Fabri (Conseil National de la Recherche, Italie), et organisée par Tania Munz (Université de Berne). Daniel Kettiger et Jean-Michel EymeriDouzans (président de l'EGPA) ont introduit la conférence, dont les sessions ont été dirigées par Andreas Lienhard et Daniel Kettiger. ${ }^{1}$

[2] La première session portait sur les aspects institutionnels et organisationnels de l'administration de la justice durant la crise du Covid-19. Durant cette session, trois contributions se sont penchées sur la réaction des tribunaux à cette situation inédite, en particulier concernant l'établissements de nouvelles règles de fonctionnement et les relations avec le pouvoir politique.

[3] Ragna Aarli (Université de Bergen, Norvège) et Wiggo Storhaug Larssen (Association des juges norvégiens, Cour d'appel de Gulating) ont inauguré la journée d'étude en proposant un regard croisé académique et praticien sur la situation en Norvège et au Danemark. Selon ces intervenant-e.s, l'indépendance du pouvoir judiciaire est l'enjeu majeur qui a pesé sur le travail de la justice au cours de la crise sanitaire. La question de savoir qui a le droit d'ordonner au président.e.s des tribunaux des modifications dans les modalités d'administration de la justice (par ex. reports dans le traitement des cas) s'est en effet posée avec acuité dans le cadre de l'épidémie de Covid-19. En Norvège, la discussion a beaucoup porté sur les risques d'infection à éviter aux professionnel-le·s et aux justiciables. Les décisions pratiques relatives à l'organisation du travail dans les tribunaux ont été prises après consultation de l'Institut National de la Santé Publique, à qui des lignes directrices ont été demandées. Au Danemark, la préservation de l'indépendance du judiciaire face aux décisions de l'exécutif a occupé le devant de la scène. La question d'un éventuel devoir de désobéissance des juges en cas de décisions politiques portant atteinte aux principes fondamentaux de la justice est posée par les intervenant.e.s. Enfin, le fait que les règles de fonctionnement judiciaire varient actuellement d'une cour à l'autre au sein d'un même pays dans le cadre de la crise pose également des problèmes en termes d'égalité d'accès à la justice. La situation aura ainsi été l'occasion de réfléchir à la préservation des principes fondamentaux du droit dans un contexte d'urgence.

[4] Costas Popotas (Cour de justice de l'Union européenne) a offert un aperçu de la manière dont le système judiciaire de l'Union européenne avait fait face à la situation. Contrairement à de nombreux pays, le système judiciaire européen disposait d'un plan d'urgence qui avait été établi à la suite d'autres crises sanitaires (scandale de l'amiante, SARS). Un comité de gestion de crise a ainsi pu être mis en place suite à l'émergence du Covid-19 afin de prendre des décisions rapides. Un système de vidéoconférence venait par ailleurs d'être instauré en 2019 avant l'émergence du

1 Lien vers le programme de la conférence : http://www.justizforschung.ch/pdf/EGPA_PSG_XVIII_Programrs_ COVID-19_V_4-0_200902.pdf. 
Covid-19, ce qui a facilité l'adaptation aux nouvelles contraintes engendrées par la crise sanitaire. Le recours aux outils digitaux dans le fonctionnement quotidien des institutions européennes, dont les collaborateurs et collaboratrices sont basée.es dans de nombreux pays, est en effet une nécessité quelles que soient les circonstances. La réorganisation du travail a également été faite en concertation avec les autorités du Luxembourg, où les institutions de justice européennes sont localisées, de même que sur la base des recommandations médicales de l'European Centre for Disease Prevention and Control. La Cour de justice de l'Union européenne entendait déjà faire une place importante aux technologies digitales dans un futur proche, ce qui a été accéléré par les circonstances. En ce sens, les effets de l'épidémie se feront sentir de manière durable dans l'organisation du travail judiciaire. L'expérience a aussi montré l'importance de se doter d'un système de gestion de crise pour faire face à ce genre de situations amenées à se répéter à l'avenir.

[5] Guy LuRIE (Israel Democracy Institute) a quant à lui proposé une analyse des mutations des activités judiciaires durant l'épidémie en Israël. Pendant la crise, les tribunaux ont accordé la priorité aux affaires urgentes, représentant environ 30\% du total des dossiers. Plusieurs projets pilotes ont par ailleurs vu le jour, avec la dématérialisation de certaines procédures. Par ailleurs, la situation a donné lieu à d'importantes tensions entre les pouvoirs d'État. Au plus fort de la crise, le Ministère de la Justice a ordonné par décrets des fermetures partielles de tribunaux, pour des durées allant de 24 heures à une semaine. Ces décrets ont été contestés par l'Association israélienne de défense des droits civils, qui a mis en avant le fait que le Parlement n'avait pas habilité le gouvernement à adopter de telles mesures. Les médias ainsi que l'opposition soupçonnaient le Premier Ministre de tenter par là de repousser son procès pour corruption et d'éviter de faire face aux charges pesant à son encontre. Cet épisode a démontré l'importance pour le système judiciaire de donner des gages de son indépendance, en particulier en période de crise et de perte de confiance publique envers le politique. Le chercheur conclut qu'à l'avenir, des décisions aussi délicates devraient être officiellement prises de manière conjointe par l'exécutif et le judiciaire, avec l'aval du Parlement, pour un meilleur équilibre entre les pouvoirs dans les processus décisionnels.

[6] La deuxième session visait à faire le point sur les effets des innovations introduites dans le cadre de la crise sanitaire. Les trois contributions ont interrogé la réactivité et les capacités d'adaptation des institutions de justice en période d'incertitude aigue, ainsi que les conséquences de ces mutations sur le quotidien de la justice dans différentes aires géographiques.

[7] Anne Wallace et Kathy Laster (Université de Victoria, Melbourne, Australie) ont détaillé les principales innovations technologiques adoptées par les Cours de justice de l'État de Victoria en Australie durant l'épidémie et interrogé leur durabilité. L'Australie n'a pas été le pays le plus prompt à adopter les outils digitaux au sein du système judiciaire, et les chercheuses perçoivent la crise du Covid-19 comme une occasion de lever certaines barrières pour une transition vers un système de justice plus flexible. Elles estiment que le Covid-19 laissera un certain degré d'innovation technologique en héritage, l'épidémie ayant eu pour effet de former une nouvelle génération de professionnel-le.s de la justice aux outils digitaux, d'en démontrer les avantages auprès du public, et de forger une expérience concrète dans le quotidien des tribunaux. Les obstacles à la durabilité des changements opérés résident dans les moyens qui seront nécessaires pour institutionnaliser les changements dans la durée, de même que dans la force d'inertie qui peut prévaloir dans certaines institutions. Considérée sur le temps long, la force des mutations actuelles doit toutefois être relativisée, tant il est vrai que la question de l'e-justice est depuis longtemps à l'agenda et n'a pas toujours connu les avancées souvent prédites à son sujet. La situation actuelle 
reste toutefois l'occasion pour les professions judiciaires de démontrer leur leadership et leur capacité de réaction.

[8] Jessica Vitorino Martins, Marcos de Moraes Sousa et Renato Máximo Sátiro (Université fédérale de Goias, Brésil) ont mis en lumière les contraintes auxquelles le judiciaire faisait face au Brésil en cette période d'urgence. Face à l'accumulation des dossiers et au ralentissement du fonctionnement de la justice, la priorité a été donnée par les tribunaux aux affaires ayant trait à la santé, au social ainsi qu'aux violences conjugales. Par ailleurs, sur la base d'une enquête menée auprès des professionnel-le·s du droit, les auteur.e.s identifient une série d'avantages découlant de la généralisation du télétravail dans le domaine judiciaire. Il s'agit notamment de la baisse des coûts du fonctionnement de la justice, de la hausse de la productivité ainsi que d'une réduction de l'absentéisme, de même que d'un gain de flexibilité dans l'allocation des ressources humaines. D'importants défis restent au contraire à relever concernant les coûts de la transition digitale, les besoins en termes de formation continue, ainsi que les problèmes posés par la volatilité du système d'accès externe aux données et à la documentation. Au niveau individuel, les membres de la profession soulignent les difficultés rencontrées dans le cadre du travail à domicile, notamment en ce qui concerne les coûts d'installation, les risques de burnout ainsi que l'enchevêtrement des sphères privées et professionnelles. Les auteur.e.s soulignent enfin que la réaction rapide et efficace de l'ordre judiciaire pour assurer la continuité de la justice en temps de crise a fortement contrasté avec les défaillances dans la réaction politique à l'épidémie du Covid-19 au Brésil.

[9] Sur la base d'une étude menée en collaboration avec Vijay Rattan, Jyoti Rattan (Université du Panjab, Inde) a pour sa part relaté les nombreux aménagements du système judiciaire qui ont également été nécessaires pour faire face à l'épidémie en Inde. Pour ce qui est des tribunaux, la priorité a été donnée aux affaires urgentes, qu'il s'agisse de dossiers en cours ou de nouveaux cas. Cela a notamment été le cas des litiges relatifs au confinement (par ex. fermeture des écoles, basculement des sessions d'examen en ligne). L'auteure a par ailleurs apporté un regard contrasté sur les effets de la transition vers l'e-justice, usuellement présentée comme apte à accélérer la cadence du travail judiciaire. Dans les faits les outils technologiques ont suscité d'importants problèmes de connectivité - une couverture internet stable n'étant pas assurée sur l'ensemble du territoire - et ont provoqué de nombreuses interruptions et retards. Ces éléments posent avec force la question des inégalités face à la justice, notamment en termes d'accès depuis les zones rurales. Ils mettent également en évidence la nécessité de protéger les droits de la défense, tant il est vrai que la transposition digitale des procédures tend à modifier les modes d'interaction entre les juges et les parties. Enfin, la crise du Covid a eu plusieurs effets importants sur les professionnel-le.s du droit, notamment un impact négatif sur les cohortes d'étudiant.e.s, un manque à gagner substantiel pour les avocat.e.s ainsi que la constitution d'un fossé générationnel entre juges dans le cadre de la numérisation des procédures.

[10] La troisième session portait sur les technologies et procédures judiciaires qui ont caractérisé cette période de crise. Les quatre contributions se sont particulièrement intéressées au rôle de catalyseur joué par la pandémie de Covid-19 dans l'adaptation des outils au sein des tribunaux pour assurer la continuité de la justice en temps de crise. Elles ont mis en évidence les opportunités mais également les défis engendrés par la crise sanitaire, notamment en termes de digitalisation, de sécurité numérique et de communication avec le public.

[11] Eric de Formanoir (Cour de cassation de Belgique) a relaté le cas de la Belgique, dont le Parlement a conféré au Gouvernement le pouvoir de prendre des mesures concernant l'organisation de la justice et l'aménagement des procédures judiciaires dès la fin du mois de mars 2020. 
Il a souligné les trois principes fondamentaux qui ont guidé l'action de la justice tout au long de la crise, à savoir le maintien de l'indépendance du pouvoir judiciaire, le respect des droits de la défense et le respect de la Constitution. Les principaux actes gouvernementaux ont été deux décrets royaux ayant pour objet l'extension de certains délais légaux en matière civile et criminelle. La caractéristique la plus frappante de la gestion de crise a été l'usage immédiat des procédures électroniques préexistantes et prêtes à l'emploi dans le système judiciaire belge, élément qui a permis de faire face à la situation. La pandémie a permis de mettre en évidence les besoins futurs en la matière, notamment la nécessité d'établir un cadre légal autour de l'usage des vidéoconférences dans le domaine judiciaire (par ex. auditions) et de renforcer différentes fonctionnalités en ligne (notamment la possibilité d'ouvrir une procédure par voie électronique). Il a toutefois été souligné que ces développements doivent avoir lieu sous réserve de l'accord des parties et selon l'appréciation du juge.

[12] João Paulo Dias, Paula Casaleiro, Teresa Maneca lima et Conceição Gomes (Université de Coimbra, Portugal) se sont penchés sur les challenges matériels posés par la crise au Portugal, en particulier en regard de la numérisation des procédures et du maintien de la sécurité sanitaire dans les bâtiments. Les auteur.e.s ont souligné combien les conditions concrètes de travail dans les tribunaux avaient un impact sur la performance des professions judiciaires et, in fine, sur la qualité de la justice rendue. Dans le cadre d'une enquête basée sur les textes législatifs et la littérature professionnelle durant la crise, l'équipe de recherche a analysé la manière dont le système judiciaire portugais avait réagi à l'épidémie. Les principaux efforts durant la crise ont porté sur la régulation du télétravail, la réorganisation des services en face-à-face ainsi que les aménagements de délais. Les auteur.e.s identifient trois types de mesures en réaction au Covid-19 : les mesures procédurales (extension ou suspension de délais, transposition de procédures en ligne), organisationnelles (télétravail, accès à distance), et physiques (équipements des bâtiments, aménagement des espaces, aération et désinfection). L'étude conclut sur la difficulté à obtenir une bonne articulation des différentes mesures en raison de la complexité organisationnelle du système judiciaire, caractérisé par une diversité de niveaux, d'institutions et de professions. Une modernisation du système judiciaire visant à renforcer la cohérence institutionnelle et à réduire les inégalités existantes dans les conditions de travail des professions judiciaires serait dès lors nécessaire.

[13] Bridget Mary McCormack (Michigan Supreme Court, USA) a fait part des expériences menées dans l'État du Michigan, qui a rapidement recouru aux nouvelles technologies en matière de travail judiciaire et de communication avec le public. Le télétravail a été effectif dès le mois de mars 2020, et la priorité a été donnée aux procédures judiciaires à distance, encouragées et soutenues par différents moyens. Afin d'encadrer ces nouvelles pratiques, des lignes directrices et des formations ont été mises en place à destination des juges, de même que des pages internet informant la population des changements en cours. La diffusion en direct des audiences constituait déjà une pratique bien établie aux États-Unis à travers l'existence de chaînes judiciaires, et a été encore renforcée durant le Covid-19. L'occasion a par ailleurs été saisie pour expérimenter différentes innovations technologiques. Une plateforme en ligne de résolution des conflits - qui était en cours de constitution mais dont le lancement a été accéléré par la crise - a été inaugurée. Un projet pilote d'expérimentation de jury en ligne a également été mené afin de récolter des informations et des bonnes pratiques pour une éventuelle concrétisation ultérieure. D'une manière générale, la situation sanitaire a été utilisée comme une opportunité de réfléchir au renforcement de l'accès à la justice. La crise a mis en évidence des besoins particuliers, notamment une explo- 
sion des recherches concernant le droit à bénéficier d'une assurance chômage durant l'épidémie. Des outils pratiques et accessibles doivent ainsi être mis à disposition du plus grand nombre sur ces questions d'actualité.

[14] Enfin John Sorabji (University College London, UK) a fait état de la situation en Angleterre et dans le Pays de Galles, où la digitalisation de la justice constituait déjà une réforme à l'agenda depuis 2016. La discussion portait principalement sur les ouvertures de procédure par voie électronique et l'e-case management, en particulier en droit civil ainsi qu'en droit de la famille. L'espoir placé dans ces réformes réside dans l'obtention d'un système judiciaire plus rapide, moins coûteux et plus praticable. Les éventuels effets de telles réformes doivent toutefois encore être évalués. Le Covid-19 en a fourni l'occasion puisque l'épidémie a rapidement promu la digitalisation du travail judiciaire par défaut, ce qui a mené le Lord Chief Justice à qualifier la situation de "plus grand projet pilote jamais réalisé à l'échelle réelle ». L'analyse des effets de ces changements devra distinguer les différents types de situations et de procédures judiciaires, et se départir d'une vision trop enchantée de la digitalisation de la justice. Ainsi, certains résultats préliminaires font état du nombre important de problèmes techniques subis dans le cadre de procédures judiciaires en ligne, ainsi que du fait que ces dernières tendent à allonger la durée des audiences et, contrairement aux attentes, à en augmenter les coûts. De même, la satisfaction du public varie en fonction du type de procédures et de leur degré de sensibilité. Ces éléments plaident en faveur de la mise en place d'étude précises et différenciées sur les mutations en cours.

[15] D'une manière générale, la journée d'étude a mis en lumière certains impératifs cruciaux pour le maintien du rôle social et démocratique des institutions judiciaires : l'égalité d'accès et de traitement pour les justiciables, la continuité de l'institution judiciaire en temps de crise, et le maintien de la marge d'appréciation du juge face à la montée en force des nouvelles technologies. La session conclusive a été l'occasion de synthétiser les enseignements de la journée et d'esquisser quelques perspectives d'avenir.

[16] Anne Sanders (Université de Bielefeld) a souligné le caractère inédit de la disruption provoquée par l'émergence du Covid-19. Premièrement, ce dernier a agi comme un catalyseur et renforcé la place des nouvelles technologiques dans les tribunaux. La transition en direction de l'e-justice ne se saurait toutefois se faire de manière automatique, et devra être accompagné de choix explicites sur les outils et les bonnes pratiques à adopter. Deuxièmement, les événements ont tout à la fois transformé la profession judiciaire en elle-même et ses liens avec le public. Des modifications dans la perception des institutions judiciaires par la société sont à attendre à l'avenir. Troisièmement, la crise a agi comme un rappel du rôle clé du judiciaire comme gardien de l'ordre constitutionnel. Elle a mis en évidence de manière aigue l'importance du contrepoids apportée par le troisième pouvoir en cette période de renforcement considérable du pouvoir exécutif. Cette fonction ne peut toutefois être endossée activement qu'à condition que le pouvoir judiciaire s'exerce dans une indépendance pleine et effective. Marco Fabri (Conseil National de la Recherche, Italie) a volontairement adopté une perspective à contre-pied de la plupart des présentations, en mettant en avant les obstacles au changement et à la réforme du fonctionnement des organisations de justice. Il a mis en évidence la tendance à l'inertie organisationnelle et le manque de moyens matériels pour les tribunaux, et remis en question la permanence des changements adoptés durant l'épidémie. Andreas Lienhard (Université de Berne, Suisse) a finalement conclu la journée d'étude en soulignant les difficultés posées par le Covid-19 pour les institutions judiciaires. Cette situation représente néanmoins une chance pour ces dernières de prouver leurs 
capacités d'innovation. Il a ainsi rappelé les défis qui restent à relever par les tribunaux, afin que le pouvoir judiciaire puisse continuer à jouer son rôle central de garant de l'État de droit.

CÉLine Mavrot, Postdoctorante à l'University of California Los Angeles (UCLA), USA, et à l’Université de Berne, Suisse. 